\title{
Patient, companion, and oncologist agreement regarding information discussed during triadic oncology clinical interactions
}

\author{
Susan Eggly'*, Louis A. Penner', Nao Hagiwara', Richard Gonzalez², Felicity W. K. Harper', Elisabeth I. Heath' and \\ Terrance L. Albrecht' \\ 'Department of Oncology, Wayne State University, Detroit, MI, USA \\ ${ }^{2}$ Department of Psychology, University of Michigan, Ann Arbor, MI, USA
}

*Correspondence to: Department of Oncology, Wayne State University, 4100 John R., MM03CB, Detroit, MI 4820I,

USA. E-mail: egglys@karmanos.org

Susan Eggly and Louis A. Penner are the primary authors.
Received: I3 September 20 I I

Revised: 5 January 2012

Accepted: 17 January 2012

\begin{abstract}
Background: Although people with cancer want and need information from their oncologists, patients and oncologists often disagree about what information was discussed during clinical interactions. Most patients have companions present during oncology visits; we investigated whether companions process information more accurately than patients. Specifically, we examined whether patients and companions differed in agreement with oncologists about what was discussed. We also investigated the effect of topic on agreement and patient/companion selfreported understanding of discussions.

Methods: Patients with companions were invited to participate on first visits to a cancer center in Detroit, MI. Patients, companions, and oncologists independently completed questionnaires immediately following visits. Participants were asked whether five topics were discussed (diagnosis, prognosis, metastasis, treatment/treatment goals, and side effects) and, if discussed, what oncologists said. Participants were also asked to estimate their own and each other's understanding of discussions.

Results: A total of 66 patient-companion-oncologist triads participated. Agreement was higher regarding whether topics were discussed than what oncologists said. Agreement did not differ by dyad type. Patients, companions, and oncologists were equally likely to be the source of triadic disagreements. Agreement was high about diagnosis $(>90 \%)$ but much lower about other topics, particularly side effects. Patients and companions reported greater understanding of discussions than oncologists estimated and more accurately estimated each other's understanding than did oncologists.

Conclusions: Companions and patients showed similar levels of agreement with oncologists about what they discussed during visits. Interventions are needed to improve communication of information to both patients and companions, especially about particular topics. Copyright () 2012 John Wiley \& Sons, Ltd.
\end{abstract}

Keywords: patient-physician communication; oncology; information exchange; family

\section{Introduction}

Most people with cancer want as much information as possible during cancer interactions [1,2], and their preferred information source is a physician or other health care provider [3-6]. Exchanging information effectively during cancer clinical interactions is associated with positive outcomes for patients [1,5,7-9]. This complex communication process, however, is only effective to the extent that patients and physicians agree about what information they discussed and have a shared understanding of key aspects of discussions [10-12]. Ineffective information exchanges can leave patients confused, dissatisfied, and potentially ill-prepared to make appropriate decisions and fully participate in their care [8,9,13-17].

Unfortunately, patients with cancer often disagree with their oncologists about what they discussed during their clinical visits, and levels of agreement vary based on the topic under discussion [15,18-20]. For example, patients and oncologists generally agree on whether they discussed a patient's diagnosis, but are less likely to agree on other topics, such as treatment goals and side effects. This disagreement may be, in part, related to patients' difficulty in processing information accurately and efficiently due to high levels of cancerrelated anxiety and distress [21,22]. Thus, having a companion present in the interaction may be helpful to patients, especially during initial meetings with an oncologist.

In fact, many patients have a companion with them when they see their physicians, particularly if they are older, sicker, and less educated [23,24]. Research shows companions provide a benefit during clinical visits by providing social and emotional support to patients and 
facilitating some aspects of information exchange between patients and physicians, such as asking questions and providing information [12,24-28]. Possibly, companions facilitate information exchange during cancer visits in other ways as well. Relative to patients, companions may experience less cancer-related anxiety and distress and are thus better able to accurately process information discussed with oncologists. If this is true, companions should show greater agreement with oncologists about what was discussed than patients and thus potentially serve as an important resource to patients as they make decisions and take actions related to their care.

Another possibility is that patient-oncologist disagreement about what information was discussed is related to oncologists' difficulties in communicating complex, high-stakes information clearly and effectively, rather than patients' inability to process information. If this is true, patients and companions should show comparable levels of agreement with oncologists about what was discussed, and companions may be less able to serve as a resource to patients following clinical visits.

To investigate these two possibilities, we examined relative levels of objective agreement about (a) whether a topic was discussed and (b) the content of the discussion (i.e., what the oncologist said about the topic) between and among patients $(\mathrm{P})$, companions $(\mathrm{C})$, and oncologists $(\mathrm{O})$ in triadic clinical interactions. We also examined whether the topic being discussed affected agreement. Additionally, we examined relative levels of patient and companion self-reported understanding of information discussed and compared them with the other participants' estimates of their understanding.

Findings from this study may inform the development of interventions to improve communication in this context. If companions show greater agreement with oncologists about what information was discussed during clinical visits than the patients they accompany, interventions should be designed to promote the role of companions in the interactions. On the other hand, if companion-oncologist agreement is similar to patientoncologist agreement, interventions should be designed to improve the way cancer health information is discussed with both patients and their companions.

\section{Participants and methods}

\section{Participants}

We conducted this study in the multidisciplinary outpatient clinic of the Karmanos Cancer Center, a National Cancer Institute-designated comprehensive cancer center in Detroit, MI, from May to August 2009. The Institutional Review Board at Wayne State University approved all study procedures. Oncologists who primarily treated solid tumors $(N=8)$ were asked to participate if they regularly saw new patients in the clinic where recruitment occurred; all 8 agreed to participate. Oncologists provided blanket consent to recruit their eligible patients. English-speaking patients of these oncologists were recruited immediately following their first visit if they had a companion with them who was present during the visit.

\section{Measures}

Oncologists, patients, and companions independently completed questionnaires about five topics routinely discussed during initial cancer clinical interactions: cancer diagnosis, prognosis, metastasis, treatment/ treatment goals, and side effects. The patient questionnaire is presented in Table 1; companion and oncologist questionnaires were adapted accordingly. Questionnaires were administered immediately after the visit to ensure participants did not communicate with each other or anyone else prior to completing them. In the first section of the questionnaire, participants were asked if each topic was discussed; if they responded affirmatively, they were also asked about the content (i.e., what the oncologist said about the topic). The only major difference in questions about topic and content was that participants were first asked if treatment was discussed (topic); if

Table I. Patient questionnaire

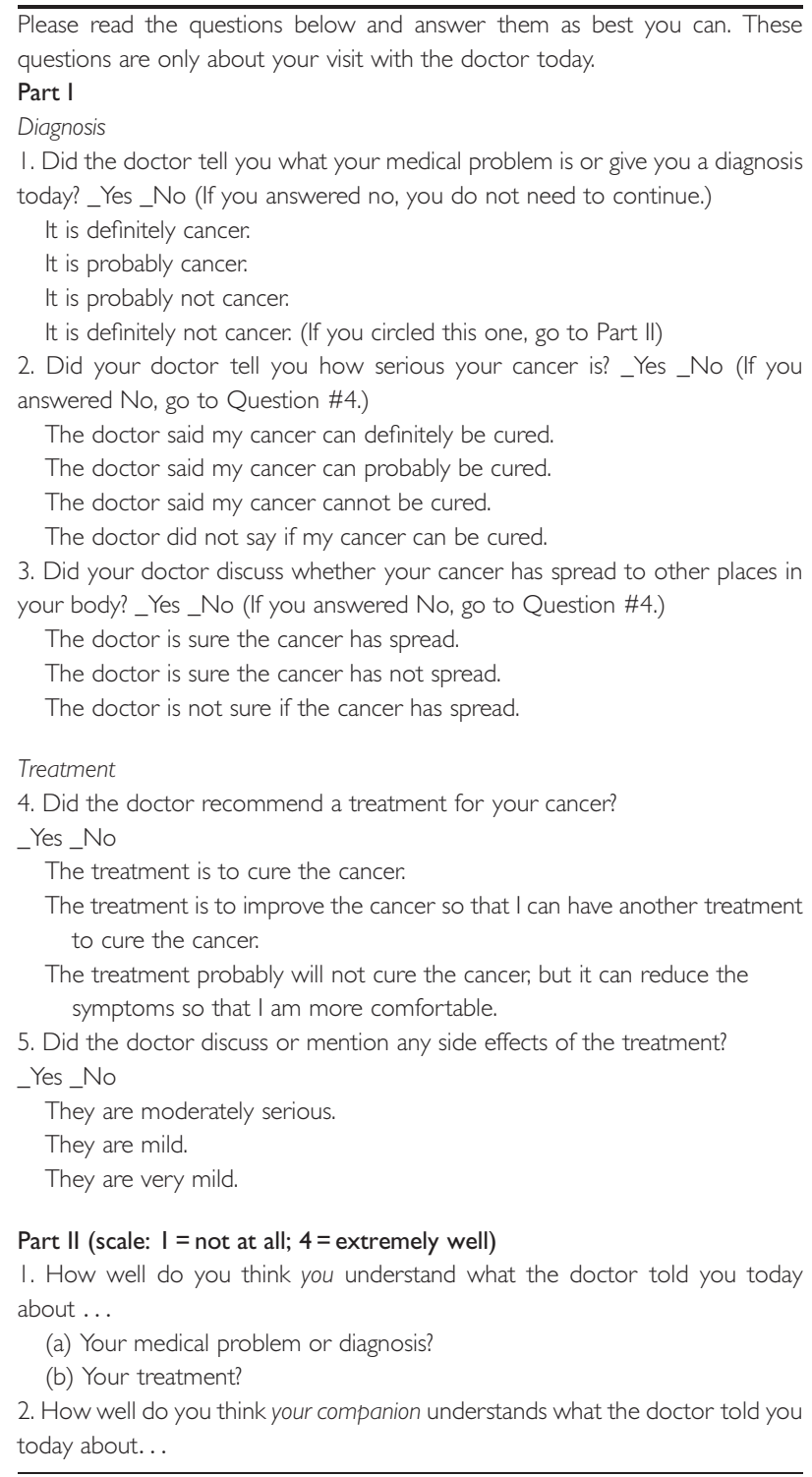


they responded affirmatively, they were asked about treatment goals (content). As the questionnaire instructions show, skip patterns were used. For example, if participants reported the topic diagnosis was discussed, they were also asked about the content (i.e., what the oncologist said about diagnosis). However, if they said diagnosis was not discussed, they were instructed to skip all remaining questions and were not included in any further analyses. This and other skip patterns resulted in substantially different sample sizes for each analysis.

In the second section, patients and companions used a four-point scale ( $1=$ 'not at all' to $4=$ 'extremely well') to rate their own and the other participant's (i.e., patient or companion) understanding of what the oncologist said about diagnosis and treatment. Using the same scale, oncologists were asked to estimate patients' and companions' understanding of these two topics. All participants also provided socio-demographic information.

\section{Analysis}

We conducted either multiple regressions (for continuous outcomes) or logistic regressions (for dichotomous outcomes) using general estimating equations (GEE) to assess associations among the variables of interest. GEE was employed because patients and companions were nested within oncologists. GEE provides consistent and asymptotically normal estimates regardless of how strongly observations within groups are correlated with one another [29]. In the logistic regression analyses, the topic 'diagnosis' was not included because participants agreed almost perfectly on this topic and thus no variability was observed. Where appropriate, contingency table analyses were used to analyze agreement/ disagreement across participants.

\section{Results}

\section{Participants}

One hundred and fifty new patients were identified during the study period. Sixty-two were not approached for the following reasons: they were unaccompanied $(n=36)$, were deemed by the oncologist to be too ill or overwhelmed to be approached $(n=21)$, or did not speak English $(n=5)$. Of the 88 remaining patient-companion dyads, $66(75 \%)$ agreed to participate and comprise the final sample. Thus, the maximum number of dyads in each analysis was 198 (i.e., three per interaction) and the maximum number of triads was 66 (one per interaction). If one member of a dyad/triad did not answer a question, the dyad/triad was not included in the analyses.

Most of the oncologists were men (87.5\%); half selfidentified as White and the others as either Asian or Middle-Eastern. The majority (75\%) had practiced for over 6 years (post-fellowship) at the cancer center. Oncology specialties included breast, gastrointestinal, genitourinary, thoracic, and malignant hematology. All but one oncologist participated in multiple interactions (range 1-17).
Patient and companion demographics are reported in Table 2. Patients were older than companions $(p<0.05)$, had higher levels of education $(p<0.001)$, and were more likely to be male $(p<0.01)$. Most companions were spouses $(56.1 \%)$. None of the patients' and companions' socio-demographic characteristics were significantly associated with the indices of agreement/ disagreement or self-reports of understanding.

\section{Dyadic and triadic agreement about whether topics were discussed}

\section{Dyads}

Figure 1 shows percent agreement for each dyad type $(\mathrm{P}-\mathrm{O}, \mathrm{C}-\mathrm{O}$, and $\mathrm{P}-\mathrm{C})$ about whether topics were discussed. Overall, $81.3 \%$ of dyads agreed about whether topics were discussed. In general, participants were more likely to agree that topics were discussed rather than were not discussed. An analysis of differences in agreements across the three dyad types showed that average agreement for $\mathrm{P}-\mathrm{O}$ was $81.7 \%$ (range: $100 \%$ for diagnosis to $62 \%$ for side effects); for $\mathrm{C}-\mathrm{O}, 78.8 \%$ (range: $98.5 \%$ for diagnosis to $64.5 \%$ for prognosis); and for $\mathrm{P}-\mathrm{C}, 83.5 \%$ (range: $98.5 \%$ for diagnosis to $74.6 \%$ for prognosis).

The GEE logistic regressions showed no significant differences among dyad types in overall percent agreement; in other words, companion-oncologist and patient-oncologist agreements were equivalent. However, regressions of four topics (excluding diagnosis because of absence of variance) revealed significant differences among topics in overall percent agreement. Specifically, the average percent agreement for side effects $(70.8 \%)$ was significantly lower than for

Table 2. Patient and companion characteristics

\begin{tabular}{|c|c|c|}
\hline Characteristics & Patients $(n=66)$ & Companions $(n=66)$ \\
\hline Mean age (SD) & $58.6(12.5)$ & $54.6(10.1)$ \\
\hline \multicolumn{3}{|l|}{ Gender } \\
\hline Male & $34(51.5 \%)$ & I 8 (27.3\%) \\
\hline Ethnicity & (Missing $=1)$ & \\
\hline Caucasian & $50(76.9 \%)$ & $50(75.8 \%)$ \\
\hline African American & II (16.9\%) & $15(22.7 \%)$ \\
\hline Asian & $3(4.6 \%)$ & | (I.5\%) \\
\hline Other & I (I.5\%) & - \\
\hline Marital status & (Missing = I) & \\
\hline Married & $46(70.8 \%)$ & $52(78.8 \%)$ \\
\hline Divorced & $4(6.2 \%)$ & $5(7.6 \%)$ \\
\hline Widowed & $5(7.7 \%)$ & $3(4.5 \%)$ \\
\hline Domestic partner & | (I.5\%) & I (I.5\%) \\
\hline Never married & $9(13.8 \%)$ & $5(7.6 \%)$ \\
\hline Annual household income (\$) & (Missing = 6) & (Missing =9) \\
\hline Less than 10,000 & $5(8.3 \%)$ & $5(8.8 \%)$ \\
\hline $10,000-19,999$ & $9(15.0 \%)$ & $5(8.8 \%)$ \\
\hline $20,000-39,999$ & $13(21.7 \%)$ & $10(17.5 \%)$ \\
\hline $40,000-59,999$ & $7(11.7 \%)$ & $6(10.5 \%)$ \\
\hline $60,000-100,000$ & $14(23.3 \%)$ & $18(31.6 \%)$ \\
\hline Greater than 100,000 & $12(20.0 \%)$ & $13(22.8 \%)$ \\
\hline Education & (Missing = 2) & (Missing = I) \\
\hline Some high school & 27 (42.2\%) & $24(36.9 \%)$ \\
\hline Some college & $18(28.1 \%)$ & $29(44.6 \%)$ \\
\hline Graduate/professional school & $19(29.7 \%)$ & $12(18.5 \%)$ \\
\hline
\end{tabular}




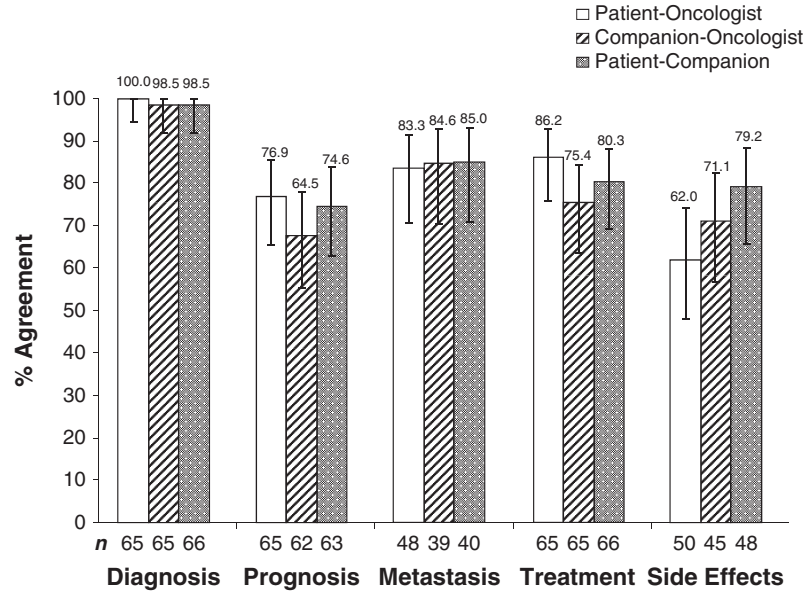

Figure I. Percent agreement by dyad type about whether topics were discussed

metastasis $\left(84.3 \% ; b=-1.06, S E=0.33, \chi^{2}(1)=10.65\right.$, $p<0.001)$ and for treatment $(80.6 \% ; b=-0.56, S E=0.26$, $\left.\chi^{2}(1)=4.64, p<0.05\right)$. There was no difference in the average percent agreement between side effects and prognosis $(72.0 \% ; p=0.60)$.

Finally, we examined the joint effects of dyad and topic on agreements about whether topics were discussed. We found no significant differences among dyad types when topics were analyzed individually.

\section{Triads}

Across the five topics, triadic agreement about whether each topic was discussed was $72.2 \%$ (Table 3). Triadic agreement was greatest for diagnosis $(98.5 \%, n=65)$ and lower for prognosis $(59.7 \%, n=62)$, metastasis $(75.0 \%, n=36)$, treatment $(70.8 \%, n=65)$, and side effects $(57.1 \%, n=42)$.

We examined which participant was the source of triadic disagreements. Of the 72 triadic disagreements across topics, oncologists were the source of $38.9 \%$ of disagreements, companions $38.9 \%$, and patients $22.2 \%$. A goodness-of-fit chi-square, comparing these percentages, was not significant $\left(\chi^{2}(2)=4.00, n s\right)$.

Finally, we considered the joint effects of triad member and topic on patterns of triadic agreement. Companions were most frequently the source of triadic disagreements for prognosis $(44.0 \%$ of 25 disagreements, where a disagreement is the sum of non-gray cells in Table 3 ) and treatment (52.6\% of 19 disagreements). Oncologists were most frequently the source of disagreements for side effects (55.6\% of 18 disagreements). However, chi-square analyses of disagreement patterns were not significant. That is, a uniform distribution of $33 \%$ disagreements for each triad member could not be rejected.

\section{Dyadic and triadic agreement about the content of discussions}

The analyses that follow are based on the subset of cases in which both dyad and all triad members agreed that a topic had been discussed and responded to questions about the content of discussions. We recorded participants' choices into dichotomized outcomes (e.g., 'definitely' and 'probably' cancer were recorded as 'cancer'; 'probably not' and 'not' cancer were recorded as 'not cancer'). Thus, in the analyses that follow, agreement does not necessarily reflect exact agreement from among the alternative responses.

\section{Dyads}

Figure 2 shows percent agreement for each dyad type ( $\mathrm{P}-\mathrm{O}, \mathrm{C}-\mathrm{O}$, and $\mathrm{P}-\mathrm{C})$ about the content of topics discussed. Overall, $62.3 \%$ of dyads agreed about content. Agreement for $\mathrm{P}-\mathrm{O}$ was $58.3 \%$ (range: $93.4 \%$ for diagnosis to $37.5 \%$ for side effects); for $\mathrm{C}-\mathrm{O}, 59.3 \%$ (range: $98.3 \%$ for diagnosis to $30.4 \%$ for side effects); and for $\mathrm{P}-\mathrm{C}, 69.1 \%$ (range: $91.9 \%$ for diagnosis to $48.9 \%$ for treatment goals).

Consistent with findings for whether topics were discussed, logistic regressions revealed no significant differences in percent agreement by dyad type; again, companion-oncologist and patient-oncologist agreements regarding content were comparable. Logistic regressions for the four topics (again excluding diagnosis) did reveal significant differences in percent agreement as a function of the content of the topics. Again, consistent with findings for whether topics were discussed, percent agreement on the content of side effects discussions $(43.2 \%)$ was significantly lower than prognosis $\left(59.8 \% ; \quad b=-1.91, \quad S E=0.48, \quad \chi^{2}(1)=15.82\right.$, $p<0.001)$ and metastasis $(67.5 \% ; b=-0.82, S E=$ $\left.0.32, \chi^{2}(1)=6.75, p<0.01\right)$. However, there was no difference between side effects and treatment goals $(59.8 \% ; p=0.38)$.

Finally, we considered the joint effects of dyad type and topic on agreement about content. We found that for side effects, $\mathrm{P}-\mathrm{C}$ agreement was significantly higher than agreement for both $\mathrm{P}-\mathrm{O}(b=1.21, S E=0.58$, $\left.\chi^{2}(1)=4.35, p<0.05\right)$ and $\mathrm{C}-\mathrm{O}(b=1.62, S E=0.59$, $\left.\chi^{2}(1)=2.77, p<0.01\right)$ agreement. There were no differences in agreement by dyad type for prognosis, metastasis, or treatment goals.

\section{Triads}

Across the five topics, $73.8 \%$ of triads agreed on content (Table 4). Agreement was highest for diagnosis $(98.3 \%, n=59)$, followed by prognosis $(94.7 \%, n=9)$, metastasis $(76.0 \%, n=5)$, treatment goals $(50.0 \%$, $n=28)$, and side effects $(50.0 \%, n=22)$.

An examination of the source of triadic disagreements about content showed that, of the 33 disagreements across topics, oncologists were the source in $45.5 \%$ of the disagreements, patients in $30.3 \%$, and companions in the remaining $24.2 \%$. A goodness-offit chi-square, comparing these percentages, was not significant $\left(\chi^{2}(2)=2.36, n s\right)$.

Finally, we considered the joint effects of triad member and topic on patterns of triadic disagreement about 
Table 3. Patterns of triadic agreement about whether topics were discussed

\begin{tabular}{|c|c|c|c|c|c|c|c|}
\hline \multicolumn{8}{|c|}{ Diagnosis (Overall triadic agreement $=98.5 \%, N=65$ ) } \\
\hline & \multicolumn{3}{|c|}{ Oncologist $=$ Yes } & & \multicolumn{3}{|c|}{ Oncologist $=$ No } \\
\hline & & \multicolumn{2}{|c|}{ Companion } & & & \multicolumn{2}{|c|}{ Companion } \\
\hline & & Yes & No & \multirow{3}{*}{ Patient } & & Yes & No \\
\hline \multirow{2}{*}{ Patient } & Yes & 64 & 1 & & Yes & 0 & 0 \\
\hline & No & 0 & 0 & & No & 0 & 0 \\
\hline \multicolumn{8}{|c|}{ Prognosis (Overall triadic agreement $=59.7 \%, N=62$ ) } \\
\hline \multirow{5}{*}{ Patient } & \multicolumn{3}{|c|}{ Oncologist $=$ Yes } & \multirow{5}{*}{ Patient } & \multicolumn{3}{|c|}{ Oncologist $=$ No } \\
\hline & & \multicolumn{2}{|c|}{ Companion } & & \multirow[b]{3}{*}{ Yes } & \multicolumn{2}{|c|}{ Companion } \\
\hline & & Yes & No & & & Yes & No \\
\hline & Yes & 36 & 10 & & & 4 & 1 \\
\hline & No & 4 & 5 & & No & 1 & 1 \\
\hline \multicolumn{8}{|c|}{ Metastasis (Overall triadic agreement $=75.0 \%, N=36$ ) } \\
\hline \multirow{5}{*}{ Patient } & \multicolumn{3}{|c|}{ Oncologist $=$ Yes } & \multirow{5}{*}{ Patient } & \multicolumn{3}{|c|}{ Oncologist $=$ No } \\
\hline & & \multicolumn{2}{|c|}{ Companion } & & \multirow[b]{3}{*}{ Yes } & \multicolumn{2}{|c|}{ Companion } \\
\hline & & Yes & No & & & Yes & No \\
\hline & Yes & 27 & 3 & & & 3 & 3 \\
\hline & No & 0 & 0 & & No & 0 & 0 \\
\hline \multicolumn{8}{|c|}{ Treatment (Overall triadic agreement $=70.8 \%, N=65$ ) } \\
\hline & \multicolumn{3}{|c|}{ Oncologist $=$ Yes } & & \multicolumn{3}{|c|}{ Oncologist $=$ No } \\
\hline & & & & & & Co & \\
\hline & & Yes & No & & & Yes & No \\
\hline Patient & Yes & 42 & 8 & Patient & Yes & 5 & 0 \\
\hline वatrot & No & 3 & 1 & alicin & No & 2 & 4 \\
\hline & & ffects & all tri & greement & $57.1 \%$ & $=42)$ & \\
\hline & & logis & & & & ologis & \\
\hline & & & & & & Co & \\
\hline & & Yes & No & & & Yes & No \\
\hline Patient & Yes & 23 & 1 & Patiti & Yes & 9 & 5 \\
\hline rallent & No & 0 & 1 & Pallent & No & 2 & 1 \\
\hline
\end{tabular}

Cases in which all three members agreed (i.e., 'Yes-Yes-Yes' or 'No-No-No') are highlighted in gray. $N=$ number of triads for each topic.

content. For treatment goals, patients were most frequently the source of disagreements $(42.9 \%$ of 14 disagreements), but for side effects, oncologists were most

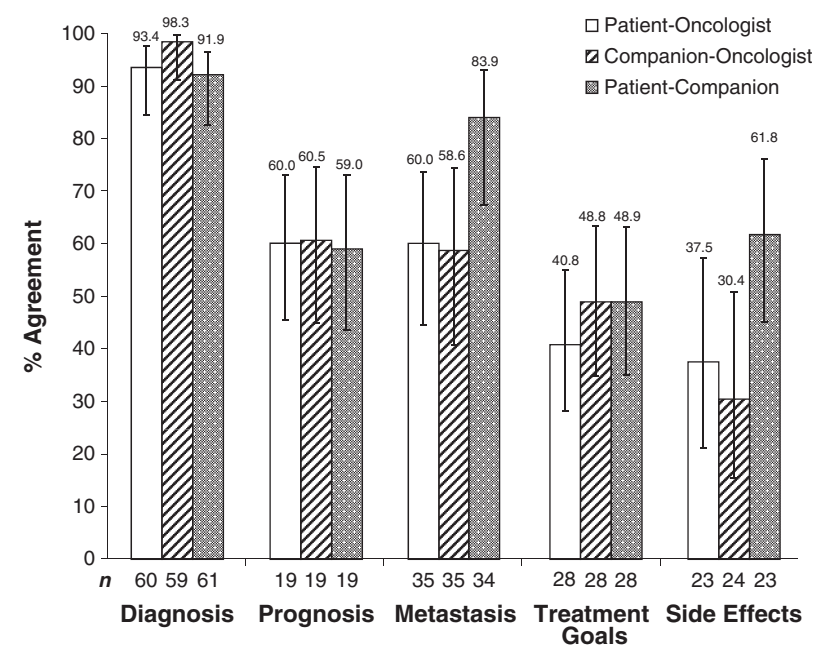

Figure 2. Percent agreement by dyad type about the content of topics discussed frequently the source ( $72.7 \%$ of 11 disagreements); the latter pattern is significantly different from a uniform distribution $\left(\chi^{2}(2)=8.91, p<0.01\right)$. There was no clear source of disagreements for the other topics. Given the very small number of disagreements about some topics, both null and significant findings must be interpreted with caution.

Self-reported and estimated understanding of patients' and companions' understanding of information

Table 5 presents means/standard deviations for patients' and companions' self-reports of their understanding of discussions of diagnosis and treatment and the other triad members' estimates of patients' and companions' understanding. Patients reported significantly more understanding of diagnosis than treatment $(p<0.001)$; there was no such difference for companions. Oncologists' estimates of both patients' and companions' understanding were invariably lower than patients' and companions' self-reports and their estimates of one another's understanding. 
Table 4. Triadic agreement and disagreement in the content of topics discussed

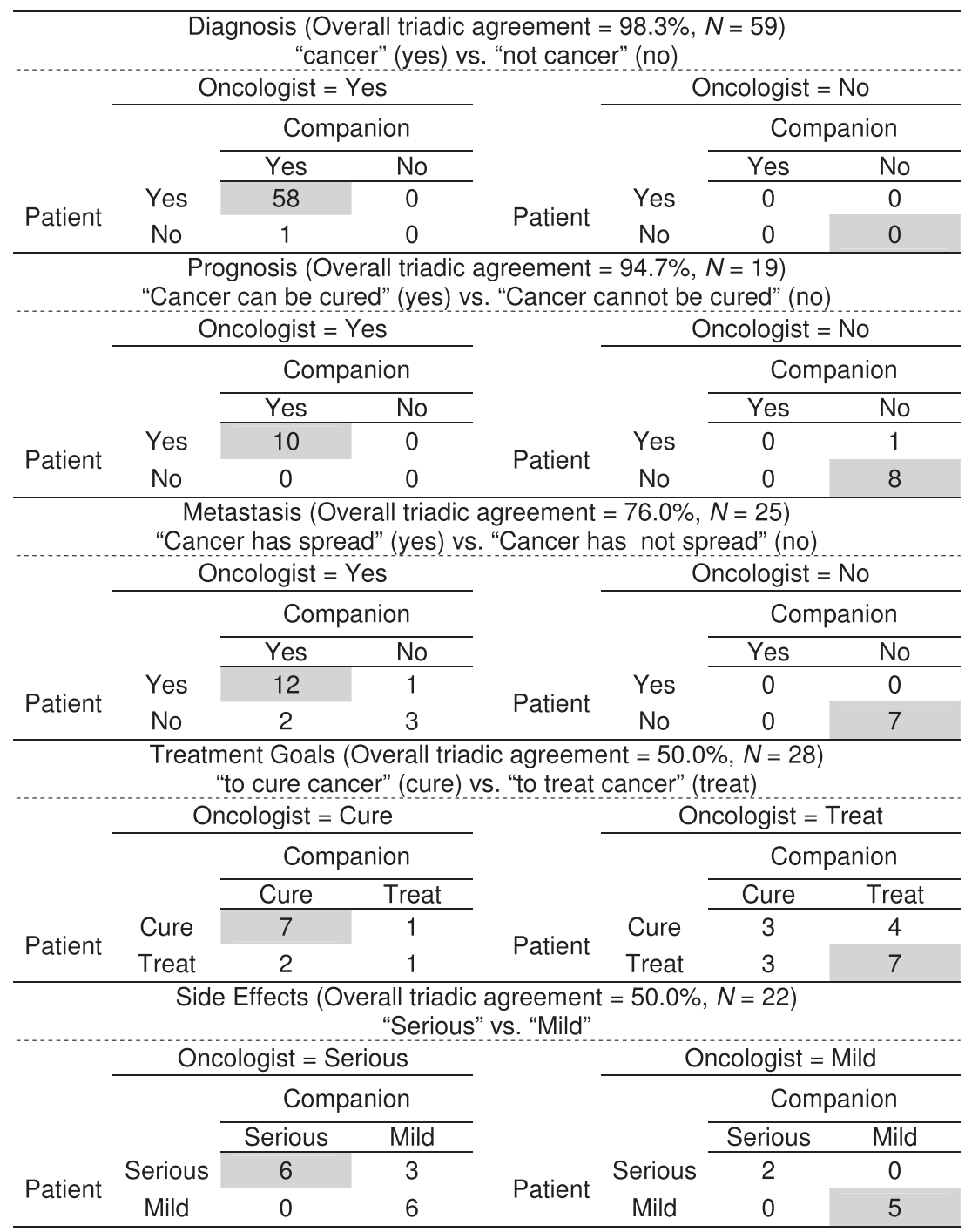

Cases in which all three members agreed are highlighted in gray. $N=$ number of triads for each topic.

Table 5. Patients' and companions' self-reported understanding of topics discussed and participants' estimates of patient and companion understanding

\begin{tabular}{lllll}
\hline Understanding & N & M & SD & b \\
\hline Patient diagnosis & 65 & 3.69 & 0.53 & \\
Companion estimate & 66 & 3.50 & 0.59 & $0.43^{* *}$ \\
Oncologist estimate & 65 & 3.34 & 0.78 & $-0.33^{*}$ \\
Patient treatment & 62 & 3.44 & 0.62 & \\
Companion estimate & 64 & 3.41 & 0.64 & $0.30 *$ \\
Oncologist estimate & 63 & 3.11 & 0.79 & 0.00 \\
Companion diagnosis & 65 & 3.60 & 0.63 & \\
Patient estimate & 65 & 3.52 & 0.62 & $0.46 * *$ \\
Oncologist estimate & 65 & 3.25 & 0.77 & -0.12 \\
Companion treatment & 62 & 3.63 & 0.52 & \\
Patient estimate & 63 & 3.40 & 0.61 & $0.39 * *$ \\
Oncologist estimate & 63 & 3.08 & 0.79 & -0.17 \\
\hline
\end{tabular}

Regression coefficients indicate the association between patient/companion selfreported understanding of diagnosis/treatment and other participants' estimate of their understanding.

*Indicates $p<0.05$

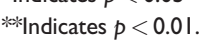

As shown in Table 5, across all four GEE regressions, there were significant positive associations between patients' and companions' estimates of one another's understanding of diagnosis and treatment. In contrast, the regression weights for the oncologists' estimates were non-significant in three of the equations and significant and negative in the equation involving patients' understanding of diagnosis. However, the significance of this regression weight may reflect a form of cooperative suppression [30] rather than a simple negative bivariate association. The bivariate association between oncologists' and companions' estimates of patients' understanding, while also negative, was actually small and non-significant $(r=-0.08)$.

\section{Discussion}

Patients and oncologists often disagree about what information they discussed during cancer interactions and potentially misunderstand key aspects of their 
discussions [15,18-20,22]. Prior research shows many patients have a family member or other companion with them in the interactions and that these companions improve some aspects of communication, by, for example, asking questions on behalf of patients [23,24,26,28]. We investigated whether companions also process information more accurately than patients in this setting. We compared companion-oncologist and patient-oncologist agreements about what information was discussed during triadic clinical interactions. We also investigated whether the topic under discussion (i.e., diagnosis, prognosis, metastasis, treatment/treatment goals, and side effects) affected levels of agreement and patient and companion self-reported understanding of topics discussed.

We found levels of agreement across dyads in these clinical interactions to be relatively high (approximately $82 \%$ ) with regard to whether a topic was discussed, but lower regarding the content of discussions (approximately $62 \%$ ). However, agreement did not differ by dyad type; in other words, companion-oncologist agreement was no higher than patient-oncologist agreement. Further, the source of disagreements within triads was fairly evenly distributed across patients, companions, and oncologists. These findings suggest companions' processing of information in this setting is no more accurate than patients'.

Consistent with prior research [15,18-20], our findings showed the topic under discussion influenced agreement. Participants generally agreed on whether diagnosis was discussed and, if discussed, what the oncologist said. Possibly, agreements were high about diagnosis because these interactions occurred in a cancer center, where presumably patients arrive having already received a diagnosis of cancer. Alternatively, if patients received a diagnosis for the first time during these interactions, their reaction to the news may have inhibited their ability to accurately process information about other topics discussed later in the visit. In fact, participants were much less likely to agree about other topics, particularly side effects. A clear understanding of these topics is critical to patients' willingness and ability to adhere to treatment recommendations. Thus, these findings suggest the need for improvement in the communication of information about most cancer topics.

We did not audio-record or video-record the interactions and thus could not determine which participant was objectively 'accurate.' Clearly, such information is valuable in analyses of patient-physician communication. However, this study focused on assessing agreement in participants' beliefs about what was discussed, rather than on comparing their beliefs with the assessment of external raters. It is participants' beliefs about what was discussed that influence decisions about treatment, and differences in these beliefs that may lead to confusion, dissatisfaction, and poorer care.

Finally, we found that patients' and companions' estimates of each other's self-reported understanding were more accurate than oncologists' estimates of patients' and companions' understanding. As mentioned previously, we measured perceived understanding rather than actual comprehension and thus cannot speculate as to the accuracy of any participant's perception. However, this finding suggests oncologists are less attuned to patients' and companions' levels of understanding than patients and companions are of each other's. This is not surprising given patients' and companions' ongoing relationship. More surprising is that the oncologists underestimated, rather than overestimated, patients' and companions' perceived understanding. Possibly, this finding indicates that oncologists are aware of the difficulties inherent in meeting the complex, high-stakes informational needs of both patients and companions in this setting. This finding, then, also suggests that interventions are needed to improve the way information is discussed with both patients and companions.

Although we collected data in a cancer center providing care to a diverse population of patients, we found no significant differences in agreement or understanding by patient socio-demographic characteristics. This is surprising given other research showing racial differences in the quality of communication in oncology interactions [25,28,31,32]. In a separate analysis of these data, we found that oncologists were significantly less likely to discuss side effects with Black patients than with White patients [33] (suggesting another aspect of the difficulties related to discussing side effects). The present study included relatively few Black patients, in part because they were more often unaccompanied than Whites and thus were ineligible. Thus, analyses of Black-White differences were generally underpowered, which could result in null findings for racial differences.

We acknowledge several other potential limitations. We did not assess non-English-speaking patients or those whose oncologists deemed them too ill or overwhelmed to participate. Patients and oncologists were more likely to be male, whereas companions were more likely to be female, which may have resulted in some residual gender effects. Future studies assessing triadic interactions would benefit from recruiting a larger and more diverse sample of patients. Future studies might also compare patient-oncologist agreement following clinical interactions in which patients are accompanied and unaccompanied.

Taken together, our findings suggest the potential for ineffective communication even when patients bring companions to clinical visits. Interventions are needed to improve this critically important aspect of patient care [34]. Some oncologists are ineffective communicators and can improve their skills through better training and modeling [35-38]. Others are highly skilled communicators, but the task of discussing complex, high-stakes information with patients and families is daunting [39]. One solution may be to have nurses, health educators, or patient advocates routinely present during clinical interactions and available to continue discussions as needed. By being present during clinical interactions with oncologists, these providers may be viewed as endorsed by oncologists. They could thus 
be an integral part of the longer-term information exchange process, reducing the potential for miscommunication that may occur if information is provided in disjointed or fragmented conversations across health care providers. Another suggestion is to provide audio recordings or written summaries of consultations, which may benefit patients and also companions not present during visits [40]. These types of interventions have the potential to improve care for all patients, whether or not companions are present during clinical interactions.

\section{Acknowledgements}

We gratefully acknowledge the support of Anthony Shields, $\mathrm{MD}, \mathrm{PhD}$ and Shirish Gadgeel, MD, who provided assistance in the development of measures; the Karmanos Cancer Institute Behavioral and Field Research Core, which provided assistance with data collection and management; and the patients, companions, and oncologists who participated in the research. This research was supported by an internal grant from the Karmanos Cancer Institute.

\section{Conflict of interest}

Authors have no financial disclosures.

\section{References}

1. Hack TF, Degner LF, Parker PA: The communication goals and needs of cancer patients: a review. Psycho-Oncology 2005; 14(10):831-845; discussion 846-837.

2. Hagerty RG, Butow PN, Ellis PM, Dimitry S, Tattersall MH: Communicating prognosis in cancer care: a systematic review of the literature. Ann Oncol 2005; 16(7):1005-1053.

3. Hesse BW, Arora NK, Burke Beckjord E, Finney Rutten LJ. Information support for cancer survivors. Cancer 2008; 112(11 Suppl):2529-2540.

4. Hesse BW, Nelson DE, Kreps GL et al. Trust and sources of health information: the impact of the Internet and its implications for health care providers: findings from the first Health Information National Trends Survey. Arch Intern Med 2005; 165(22):2618-2624.

5. Rutten LJ, Arora NK, Bakos AD, Aziz N, Rowland J. Information needs and sources of information among cancer patients: a systematic review of research (1980-2003). Patient Educ Couns 2005; 57(3):250-261.

6. Arora NK, Hesse BW, Rimer BK, Viswanath K, Clayman ML, Croyle RT. Frustrated and confused: the American public rates its cancer-related information-seeking experiences. Gen Intern Med 2008; 23(3):223-228.

7. Arora NK. Interacting with cancer patients: the significance of physicians' communication behavior. Soc Sci Med 2003; 57(5):791-806.

8. Griggs JJ, Sorbero ME, Mallinger JB et al. Vitality, mental health, and satisfaction with information after breast cancer. Patient Educ Couns 2007; 66(1):58-66.

9. Epstein RM, Street RL, Jr. Patient-centered Communication in Cancer Care: Promoting Healing and Reducing Suffering. National Cancer Institute: Bethesda, MD; 2007.

10. Rogers E, Kincaid DL. Communication Networks: A Paradigm for New Research. Free Press: New York; 1981.

11. Albrecht TL, Penner LA, Cline RJ, Eggly SS, Ruckdeschel JC. Studying the process of clinical communication: issues of context, concepts, and research directions. J Health Commun 2009; 14 Suppl 1:47-56.

12. Albrecht TL, Eggly S, Ruckdeschel JC. Communicating with relatives/companions about cancer care. In Handbook of Communication in Oncology and Palliative Care. Kissane D, Bultz B, Butow P, Finlay I (eds). Oxford University Press: Oxford, UK; 2010: 157-164.

13. Mallinger JB, Griggs JJ, Shields CG. Patient-centered care and breast cancer survivors' satisfaction with information. Patient Educ Couns 2005; 57(3):342-349.

14. Ayanian JZ, Zaslavsky AM, Arora NK et al. Patients' experiences with care for lung cancer and colorectal cancer: findings from the Cancer Care Outcomes Research and Surveillance Consortium. J Clin Oncol 2010; 28(27): 4154-4161.

15. Gabrijel S, Grize L, Helfenstein E et al. Receiving the diagnosis of lung cancer: patient recall of information and satisfaction with physician communication. J Clin Oncol 2008; 26(2):297-302.

16. Dimoska A, Butow PN, Dent E, Arnold B, Brown RF, Tattersall MH. An examination of the initial cancer consultation of medical and radiation oncologists using the Cancode interaction analysis system. Br J Cancer 2008; 98(9):1508-1514.

17. Albrecht TL, Eggly SS, Gleason ME et al. Influence of clinical communication on patients' decision making on participation in clinical trials. J Clin Oncol 2008; 26(16): 2666-2673.

18. Quirt CF, Mackillop WJ, Ginsburg AD et al. Do doctors know when their patients don't?: a survey of doctor-patient communication in lung cancer. Lung Cancer 1997; 18(1): $1-20$.

19. Meropol NJ, Weinfurt KP, Burnett CB et al. Perceptions of patients and physicians regarding phase I cancer clinical trials: implications for physician-patient communication. $J$ Clin Oncol 2003; 21(13):2589-2596.

20. Jenkins V, Solis-Trapala I, Langridge C, Catt S, Talbot DC, Fallowfield LJ. What oncologists believe they said and what patients believe they heard: an analysis of phase I trial discussions. J Clin Oncol 2011; 29(1):61-68.

21. Eysenck MW, Derakshan N. New perspectives in attentional control theory. Pers Indiv Differ 2011; 50(7): 955-960.

22. Beckjord EB, Finney Rutten LJ, Arora NK, Moser RP, Hesse BW. Information processing and negative affect: evidence from the 2003 Health Information National Trends Survey. Health Psychol 2008; 27(2):249-257.

23. Wolff JL, Roter DL. Hidden in plain sight: medical visit companions as a resource for vulnerable older adults. Arch Intern Med 2008; 168(13):1409-1415.

24. Wolff JL, Roter DL. Family presence in routine medical visits: a meta-analytical review. Soc Sci Med 2011; 72(6): 823-831.

25. Street RL, Gordon HS. Companion participation in cancer consultations. Psycho-Oncology 2008; 17(3):244-251.

26. Clayman ML, Roter D, Wissow LS, Bandeen-Roche K. Autonomy-related behaviors of patient companions and their effect on decision-making activity in geriatric primary care visits. Soc Sci Med 2005; 60(7):1583-1591.

27. Eggly S, Penner LA, Greene M, Harper FW, Ruckdeschel JC, Albrecht TL. Information seeking during "bad news" oncology interactions: question asking by patients and their companions. Soc Sci Med 2006; 63(11):2974-2985.

28. Eggly S, Harper FW, Penner LA, Gleason MJ, Foster T, Albrecht TL. Variation in question asking during cancer clinical interactions: a potential source of disparities in access to information. Patient Educ Couns 2011; 82(1): 63-68.

29. Hanley JA, Negass A, Edwardes MD, Forrester JE. Statistical analysis of correlated data using generalized estimating equations: an orientation. Am J Epidemiol 2003; 157: 364-375.

30. Cohen J, Cohen P. Applied Multiple Regression/Correlation Analysis for the Behavioral Sciences. Lawrence Erlbaum Associates; distributed by Halsted Press Division of John Wiley: Hillsdale, N.J, New York; 1975. 
31. Gordon HS, Street RL, Jr., Sharf BF, Souchek J. Racial differences in doctors' information-giving and patients' participation. Cancer 2006; 107(6):1313-1320.

32. Jean-Pierre P, Fiscella K, Griggs J et al. Race/ethnicitybased concerns over understanding cancer diagnosis and treatment plan. J Natl Med Assoc 2010; 102(3):184-189.

33. Eggly S, Penner LA, Harper FW, Zhdanova L, Gonzalez R, Albrecht TL. Perceptions of information provided by oncologists in clinical interactions with black and white patients/companions. In: Proceedings of the Conference of the American Association for Cancer Research (AACR) Science of Cancer Health Disparities in Racial/Ethnic Minorities and the Medically Underserved: 2010; Miami, FL: Cancer Epidemiol. Biomarkers Prev; 2010: A9.

34. Mitnick S, Leffler C, Hood VL. Family caregivers, patients and physicians: ethical guidance to optimize relationships. $J$ Gen Intern Med 2010; 25(3):255-260.

35. Back AL, Arnold RM, Baile WF et al. Efficacy of communication skills training for giving bad news and discussing transitions to palliative care. Arch Intern Med 2007; 167(5): 453-460.

36. Bylund CL, Brown RF, Bialer PA, Levin TT, Lubrano di Ciccone B, Kissane DW. Developing and implementing an advanced communication training program in oncology at a comprehensive cancer center. J Cancer Educ 2011; 26(4): 604-611.

37. Brown RF, Bylund CL. Communication skills training: describing a new conceptual model. Acad Med 2008; 83(1): $37-44$.

38. Fallowfield L, Jenkins V, Farewell V, Solis-Trapala I. Enduring impact of communication skills training: results of a 12-month follow-up. Br J Cancer 2003; 89(8):1445-1449.

39. Back AL, Arnold RM, Baile WF, Tulsky JA, Fryer-Edwards $\mathrm{K}$. Approaching difficult communication tasks in oncology. CA Cancer J Clin 2005; 55(3):164-177.

40. Pitkethly M, Macgillivray S, Ryan R: Recordings or summaries of consultations for people with cancer. Cochrane Database Syst Rev 2008; (3):CD001539. 